\title{
Erratum: Semianalytic calculation of cosmic microwave background anisotropies from wiggly and superconducting cosmic strings [Phys. Rev. D 96, 103535 (2017)]
}

\author{
I. Yu. Rybak, A. Avgoustidis, and C. J. A. P. Martins
}

(Received 27 July 2019; published 12 August 2019)

DOI: 10.1103/PhysRevD.100.049901

(1) The definition of the Nambu-Goto action (3) should contain a factor of $1 / 2$ inside the square root so as to be consistent with the definition of $\gamma$ in Eq. (2). Thus, Eq. (3) should be rewritten as

$$
S=-\mu_{0} \int f(\kappa, \gamma, \Delta) \sqrt{-\gamma / 2} d^{2} \sigma
$$

(2) In the definitions of $\tilde{U}, \tilde{T}$, and $\Phi$ all terms with a factor of $\frac{\partial f}{\partial \kappa}$ appear with the wrong sign. In particular, Eqs. (5)-(7) should be written as

$$
\begin{aligned}
& \tilde{U}=f-2 \frac{\partial f}{\partial \gamma} \frac{\Delta}{\gamma}-2 \gamma^{00} \frac{\partial f}{\partial \kappa} \dot{\varphi}^{2}+2 \gamma^{11} \frac{\partial f}{\partial \Delta} \varphi^{\prime 2}, \\
& \tilde{T}=f-2 \frac{\partial f}{\partial \gamma} \frac{\Delta}{\gamma}-2 \gamma^{11} \frac{\partial f}{\partial \kappa} \varphi^{\prime 2}+2 \gamma^{00} \frac{\partial f}{\partial \Delta} \dot{\varphi}^{2}, \\
& \Phi=\frac{2}{\sqrt{-\gamma}}\left(-\frac{\partial f}{\partial \kappa}-\frac{\partial f}{\partial \Delta}\right) \varphi^{\prime} \dot{\varphi},
\end{aligned}
$$

and Eqs. (12)-(14) should be

$$
\begin{aligned}
& \tilde{U}=f+2 \sum_{i}\left(-\gamma^{00} \frac{\partial f}{\partial \kappa_{i}} \dot{\varphi}_{i}^{2}+\gamma^{11} \frac{\partial f}{\partial \Delta_{i}} \varphi_{i}^{\prime 2}-\frac{\partial f}{\partial \gamma} \frac{\Delta_{i}}{\gamma}\right), \\
& \tilde{T}=f+2 \sum_{i}\left(-\gamma^{11} \frac{\partial f}{\partial \kappa_{i}} \varphi_{i}^{\prime 2}+\gamma^{00} \frac{\partial f}{\partial \Delta_{i}} \dot{\varphi}_{i}^{2}-\frac{\partial f}{\partial \gamma} \frac{\Delta_{i}}{\gamma}\right), \\
& \Phi=\frac{2}{\sqrt{-\gamma}} \sum_{i}\left(-\frac{\partial f}{\partial \kappa_{i}}-\frac{\partial f}{\partial \Delta_{i}}\right) \varphi_{i}^{\prime} \dot{\varphi}_{i} .
\end{aligned}
$$

(3) In Eq. (29), (32) the " $W$ " multiplier for the momentum parameter $k(v)$ is missing, i.e., Eq. (29) should be

$$
\dot{v}+v \frac{\dot{a}}{a}\left(1-v^{2}\right)(1+W)-\left(1-v^{2}\right) W \frac{k(v)}{R_{c}}=\left\langle 2 \frac{\Phi}{\tilde{U}} \frac{1-\dot{\mathbf{x}}^{2}}{1-\tilde{T} / \tilde{U}}\left(\frac{\tilde{T}^{\prime}}{\epsilon}+2 \frac{\dot{a}}{a} \Phi+\dot{\Phi}-2 \Phi \frac{\mathbf{x}^{\prime \prime} \cdot \dot{\mathbf{x}}}{\mathbf{x}^{\prime 2}}\right)\right\rangle
$$

and Eq. (32) should be

$$
\dot{v}+v \frac{\dot{a}}{a}\left(1-v^{2}\right)(1+W)-\left(1-v^{2}\right) W \frac{k(v)}{\xi_{c}}=2 \frac{Q}{\hat{U}} \frac{1-v^{2}}{1-W}\left(\sqrt{1-v^{2}} \hat{T}_{, s}+\dot{Q}+2 Q\left(\frac{\dot{a}}{a}-\frac{k(v) v}{\xi_{c}}\right)\right) .
$$

(4) We would also like to stress the fact that $\kappa$ and $\Delta$ are not independent variables. However, it is beneficial to introduce them as they help to organize the equations into useful form, see for example Eqs. (5-7). The relation between $\kappa$ and $\Delta$ can be imposed at the end of the calculation.

These corrections do not change the results and conclusions of the paper. In particular, all plots are visually identical. We thank Patrick Peter for pointing out these issues. 